\title{
Thermomechanical Fatigue of Unidirectional Carbon Fiber/Epoxy Composite in Space
}

\author{
Ali Anvari (D) \\ Department of Mechanical and Aerospace Engineering, University of Missouri-Columbia, Columbia, Missouri, USA \\ Correspondence should be addressed to Ali Anvari; aabm9@mail.missouri.edu
}

Received 24 April 2020; Revised 9 June 2020; Accepted 16 June 2020; Published 29 June 2020

Academic Editor: Yuanxin Zhou

Copyright (๑ 2020 Ali Anvari. This is an open access article distributed under the Creative Commons Attribution License, which permits unrestricted use, distribution, and reproduction in any medium, provided the original work is properly cited.

\begin{abstract}
Thermomechanical fatigue is one of the challenges for spacecrafts during space missions. As a result of the extreme temperature variation in space, thermal cycles are created, and due to the imposed mechanical loads to spacecrafts such as engine loads while they turn on and off, mechanical cycles are created. The worst structural fatigue for spacecrafts occurs when both thermal and mechanical cycles happen, simultaneously. The reason is that both thermal and mechanical cycles could cause stress concentration in the spacecraft structure. Therefore, the probability of crack initiation or propagation in the spacecraft structure increases. In this study, for the first time, novel thermomechanical fatigue relations are introduced to evaluate the safety of unidirectional carbon fiber/epoxy composite in the space environment. This composite material has been used in the spacecraft structure due to its lightweight and high strength. Furthermore, with applying thermal fatigue relations, thermally-safe planets, moons, and asteroids in the solar system for unidirectional carbon fiber/epoxy composite are identified.
\end{abstract}

\section{Introduction}

Unidirectional Carbon Fiber-Reinforced Epoxy (UD CFRE) has been used in many applications such as aerospace, automotive, sporting goods, and ships. In the space industry, UD CFRE can be used to fabricate the spacecraft structures [1]. UD CFRE composites have been used in space application due to their best mechanical properties such as low weight and high strength. Nevertheless, one of the failure modes of UD CFRE is due to the thermomechanical fatigue in the space environment. Therefore, the effect of thermomechanical fatigue on the mechanical behavior of this material is under extensive study [2].

The application of UD CFRE is very broad in many industries. It could be applied in automobiles, tidal turbine blades, jet-engine fan blades, wind turbine blades, and structural members of an aircraft. Experiments have shown that the failure mechanism in UD CFRE could be in the form of interlaminar and intralaminar matrix cracks, fiber-matrix debonding, and fiber breakage [3]. Mechanical properties of CFRE include corrosion resistance, high strength to weight ratio, nonmagnetic properties, and fatigue resistance. Epoxy is a thermosetting resin. Therefore, its mechanical properties degrade intensely when it is exposed to elevated temperatures in the space environment. The epoxy deterioration can be severe in cases where the extreme heat in space is higher than the glass transition temperature $\left(T_{\mathrm{g}}\right)$ and the decomposition temperature $\left(T_{\mathrm{d}}\right)[4,5]$.

Design processes have shown that fatigue properties are important characteristics of engineering materials. The reason is the statistics which has indicated that fatigue failures are over $90 \%$ of all the failures. The experiments have shown that, at temperatures higher than $100^{\circ} \mathrm{C}$, fatigue strength of CFRE decreases significantly. The reduction in the fatigue strength of CFRE is due to the induced defects which causes high stress concentration [6].

Since the birth of CFRE, many studies have been provided to evaluate the mechanical properties, failure, and fatigue of this composite [7-13]. Among these studies, "significance of defects in the fatigue failure of carbon fiber-reinforced plastics" is submitted by Prakash [7]. Furthermore, "micromechanisms of interlaminar fracture in carbon-epoxy composites at multidirectional ply interfaces" is published by Singh and Greenhalgh [8]. Additionally, "prediction of tensile 
fatigue life for unidirectional CFRE, in: progress in durability analysis of composite system," is studied by Miyano et al. [9].

A few studies have been provided to predict the thermal fatigue of UD CFRE $[14,15]$ in space. Nevertheless, there is no research to predict the thermomechanical fatigue of UD CFRE. Therefore, in this study, for the first time, relations are introduced to predict the thermomechanical fatigue of UD CFRE is space. Thermomechanical fatigue occurs when a spacecraft is exposed to thermal and mechanical cycles, simultaneously. Each time the spacecraft engine turns on and off, it is both a mechanical cycle and a thermal cycle because it imposes mechanical stress on the spacecraft, and the engine heats up and cools down, simultaneously. Furthermore, each time the spacecraft passes through a shadow of a planet, moon, or an asteroid, its temperature decreases, and while it passes through the sun illumination, its temperature increases. Therefore, a thermal cycle can be created as a result of cooling down and heating up.

Stress concentration and possible crack initiation and/or propagation can develop in a spacecraft structure as a result of thermal and mechanical cycles. Hence, while thermal and mechanical cycles happen at the same time, the stress concentration which is induced in the structure and the probability of crack initiation and/or propagation in the structure could be higher than when only thermal or mechanical cycle occurs. Therefore, an exact analysis to evaluate the thermomechanical fatigue in space missions is required. Thus, in this study, thermomechanical fatigue of UD CFRE is evaluated.

\section{Problem Formulation}

In 2018 [15], it has been proved that the degradation of Interlaminar Shear Strength (ILSS) of UD CFRE is the main cause of failure in a thermal fatigue condition. The reason is due to the Interlaminar Shear stress (ILSs) within the Carbon Fiber (CF) and epoxy interface while UD CFRE is exposed to thermal cycles. The main cause of developing ILSs within the CF and epoxy interface is the difference of the Coefficient of Thermal Expansions (CTE) between these two materials in axial direction. Therefore, ILSs equation [15] has been defined as follows:

$$
\text { ILSs }=G_{\text {CF }}|(23-T)| \cdot\left|\alpha_{\text {epoxy.A }}-\alpha_{\text {CF.A }}\right| \text {. }
$$

In equation (1), $G_{C F}$ is the shear modulus of $C F$ in the axial direction, 23 is the crack-free or stress-free temperature in Celsius, $T$ is the space temperature, $\alpha_{\text {epoxy.A }}$ is the CTE of epoxy in the axial direction, and $\alpha_{\text {CF.A }}$ is the CTE of CF in the axial direction.

In a thermal cycling environment, ILSs must be less than ILSS. As a result, relation (2) for thermal fatigue is developed. In relation (2), F.S..$_{T}$ is the thermal fatigue factor of safety.

$$
\frac{\text { ILSs }}{\text { ILSS }}=\text { F.S. }_{T}<1 \text {. }
$$

For deriving a relation for thermomechanical fatigue, mechanical fatigue expression must be added to relation (2). Therefore, the first thermomechanical fatigue relation is indicated as follows:

$$
\frac{\text { ILSs }}{\text { ILSS }} \text { F.S. }{ }_{T}+\frac{\sigma_{M}}{\sigma_{f}} \text { F.S. } \cdot_{M}<1 .
$$

In relation (3), F.S. ${ }_{M}$ is the factor of safety for mechanical fatigue, $\sigma_{M}$ is the amplitude of applied mechanical stress, and $\sigma f$ is the failure mechanical stress. $\sigma_{M}$ and $\sigma_{f}$ must be both either compression or tension. For the highest safety, both compression and tension stresses must be evaluated in relation (3). The next thermomechanical relation, which must be evaluated in order to have a safe design for UD CFRE, is as follows:

$$
\frac{\left(\Delta T_{\text {space }}\right) N_{T}}{\left(\Delta T_{\text {exp. }}\right) N_{T . f}} \text { F.S. } ._{T}+\frac{\sigma_{M}}{\sigma_{f}} \text { F.S. } ._{M}<1 .
$$

In the relation (4), $\Delta T_{\exp }$. is the temperature variation at each thermal cycle in the experiment which has been conducted on UD CFRE. $N_{T . f}$ is the number of thermal cycles to failure in the thermal cyclic experiment. $\Delta T_{\text {space }}$ is the temperature variation at each thermal cycle in space. $N_{T}$ is the number of thermal cycles in space. If there are two or multiple space environments with different thermal cycles with different temperature variations at each thermal cycle, then the following relation must be used:

$$
\frac{\sum_{i=0}^{n} \Delta T_{i} \cdot \mathrm{NT}_{i}}{\left(\Delta T_{\exp .}\right) N_{T . f}} \text { F.S. } \cdot_{T}+\frac{\sigma_{M}}{\sigma_{f}} \text { F.S. }{ }_{M}<1 .
$$

ILSS, F.S.T, F.S.M ${ }$, and $N_{T . f}$ must be obtained by conducting experiments on UD CFRE. According to this thermomechanical fatigue evaluation method, relations (2)-(4) must hold to make sure that UD CFRE is fail-safe or in other words, it does not fail under thermomechanical fatigue.

Based on the latest experiments, at temperatures less than $0^{\circ} \mathrm{C}$, epoxy shows a brittle behavior. Therefore, at temperatures less than $0^{\circ} \mathrm{C}$, mechanical fatigue tensile stress amplitude must be less than $600 \mathrm{MPa}\left(\sigma_{M}<600 \mathrm{MPa}\right)$ [6]. Furthermore, the number of mechanical fatigue cycles must be less than $10^{5}\left(N_{M}<10^{5}\right)$ [2] to prevent brittle fracture. Additionally, experiments have shown that after epoxy had been exposed to thermal cycles, its glass transition temperature $\left(T_{\mathrm{g}}\right)$ has been reduced by $9 \%$ [5]. Therefore, in thermal cycling environments, the epoxy temperature must be always less than $0.91 T_{\mathrm{g}}$ to make sure it can carry load and does not fail. It seems necessary to mention that for different applications such as aerospace and automotive, the values which can be chosen for mechanical and thermal fatigue factors of safety can be different. Nevertheless, based on the results of research studies and experiments which have been conducted $[2,5,6,14,15]$, for the thermal and mechanical fatigue factors of safety in relations (2), (3), (4), and (5), the minimum value of 1.5 is recommended.

\section{Safe Planets, Moons, and Asteroids in the Solar System for UD CFRE}

In this section of the manuscript, thermal analysis is provided to identify which planets, moons, and asteroids are safe for UD CFRE in the spacecraft structure. For this purpose, the following relation is employed: 
TABLE 1: Safe planets, moons, and asteroids for UD CFRE in the inner solar system [16].

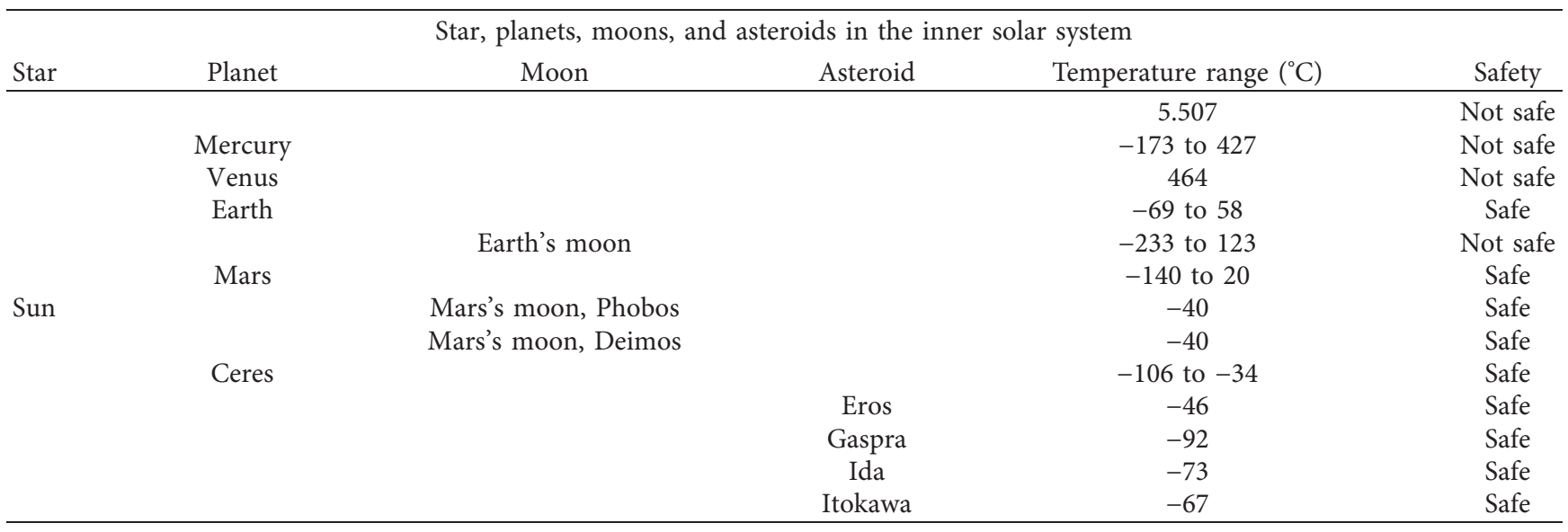

ILSS $=$ ILSs.

Relation (6) indicates that when ILSs reaches the ILSS, it is the end of the thermal life for UD CFRE. For solving relation (6), it should be extended and written in the form of relation (7). All the parameters in the relation (7) are the same as in equation (1), and the only difference is that instead of ILSs at the left-hand side of the equation, ILSS is substituted.

$$
\text { ILSs }=G_{\mathrm{CF}} \cdot|(23-T)| \cdot\left|\alpha_{\text {epoxy.A }}-\alpha_{\mathrm{CF} . A}\right| \cdot
$$

Since all the values of ILSS, $G_{\mathrm{CF}}, \alpha_{\text {epoxy.A }}$, and $\alpha_{\mathrm{CF} . A}$ are available [15]. Therefore, with substituting these values in the relation (7), the following relation is obtained:

$$
80.9 e+6=(7.59 e+9) .|(23-T)| .|(43.92 e-6)-(-0.83 e-6)| .
$$

By solving equation (8), $T$ is derived, and it has two values. $T_{\min }=-215^{\circ} \mathrm{C}$ and $T_{\max }=261^{\circ} \mathrm{C}$. It means that the temperature of UD CFRE should be between -215 and $261^{\circ} \mathrm{C}$. The reason is that if the temperature of UD CFRE reaches -215 or $261^{\circ} \mathrm{C}$, the ILSs reache the ILSS and the composite material could fail at these temperatures due to the stress concentration in the CF and epoxy interface. Hence, UD CFRE is safe in temperatures between -215 and $261^{\circ} \mathrm{C}$. However, if a mechanical load applies to UD CFRE at this temperature range, the safety must be evaluated based on the thermomechanical fatigue relations which have been introduced in the previous section. Please note that, in equation (8), ILSS and $G_{\mathrm{CF}}$ are in $\mathrm{N} / \mathrm{m}^{2}, T$ is in Celsius, and CTEs are in $1 /{ }^{\circ} \mathrm{C}$ units.

Based on this safe temperature range $\left(-215^{\circ} \mathrm{C}<T<261^{\circ} \mathrm{C}\right)$, in this part, it can be evaluated which planets, moons, and asteroids are safe for UD CFRE. According to the data in Tables 1 and 2 which are provided by Chown in 2011 [16], temperature range of planets, moons, and asteroids in the inner (Table 1) and outer (Table 2) solar system are indicated. If the temperature range is between the -215 and $261^{\circ} \mathrm{C}$, they are thermally-safe for UD CFRE.

\section{Future Work}

Due to the excellent properties of Carbon Nanotubes (CNT), nowadays, its application is broad in many industries such as aerospace and automotive industries. Carbon nanotubes have great mechanical properties such as high tensile strength, high Young's modulus, and high aspect ratio which makes CNT one of the best materials for different applications. Furthermore, the electrical conductivity of CNT is high $[17,18]$. Hence, recently, for enhancing the knowledge regarding the CNTs, a few studies to further analyze the mechanics and forest synthesis of CNT have been published [19-22]. Since CNTs have superior mechanical properties when they are compared with carbon fibers such as higher tensile strength, they are expected to be applied widely in future space structures. Therefore, for the future work, research regarding to the "thermomechanical fatigue of CNTreinforced epoxy" is recommended.

\section{Discussion}

As it has been discussed in Section 2, at temperatures less than $0^{\circ} \mathrm{C}$, epoxy is brittle, and it can crack when applying a smaller load with a much higher crack growth rate. Therefore, at temperatures less than $0^{\circ} \mathrm{C}$, the factor of safeties, which must be used in relations (2)-(5), needs to be adjusted according to epoxy brittleness. Hence, F.S. ${ }_{T}$ and F.S. ${ }_{M}$ must have higher values. However, the amount of the factor of safeties must be determined by conducting thermomechanical fatigue experiments.

Furthermore, after several hundred thermal cycles, the epoxy glass transition temperature will drop by $9 \%$. Therefore, epoxy will soften at lower temperatures and is not capable to carry load and withstand mechanical cycles. Hence, this drop in Tg must be considered in design processes and determining the appropriate values of safety factors.

All the thermomechanical fatigue relations which have been introduced in this work can be applied for carbon nanotube-reinforced epoxy as well. Nevertheless, the amounts of safety factors, maximum allowed number of thermal and mechanical cycles, and maximum ILSs, and 
TABLE 2: Safe planets, moons, and asteroids for UD CFRE in the outer solar system [16].

\begin{tabular}{|c|c|c|c|c|}
\hline Planet & Moon & Asteroid & Temperature range $\left({ }^{\circ} \mathrm{C}\right)$ & Safety \\
\hline Jupiter & & & -163 to -121 & Safe \\
\hline & Jupiter's moon, Io & & -183 to -143 & Safe \\
\hline & Jupiter's moon, Europa & & -223 to -148 & Not safe \\
\hline & Jupiter's moon, Ganymede & & -203 to -121 & Safe \\
\hline & Jupiter's moon, Callisto & & -193 to -108 & Safe \\
\hline Saturn & & & -191 to -130 & Safe \\
\hline & Saturn's moon, Titan & & -179 & Safe \\
\hline & Saturn's moon, Enceladus & & -240 to -128 & Not safe \\
\hline & Saturn's moon, Lapetus & & -173 to -143 & Safe \\
\hline & Saturn's moon, Mimas & & -209 & Safe \\
\hline & Saturn's moon, Hyperion & & -180 & Safe \\
\hline Uranus & & & -214 to -205 & Safe \\
\hline & Uranus's moon, Miranda & & -223 to -187 & Not safe \\
\hline Neptune & & & -223 to -220 & Not safe \\
\hline & Neptune's moon, Triton & & -235 & Not safe \\
\hline Pluto & & & -240 to -218 & Not safe \\
\hline Eris & & & -246 to -230 & Not safe \\
\hline Makemake & & & -243 to -238 & Not safe \\
\hline
\end{tabular}

mechanical stress must be adjusted to the mechanical and thermal properties of CNT/epoxy.

For determining the thermally-safe planets, moons, and asteroids for UD CFRE, in Section 3, it has been assumed that the UD CFRE does not have a coating against the space temperature. Thus, if an appropriate coating can be applied to cover the UD CFRE, it can enhance the safety temperature range.

\section{Conclusions}

In this research, by applying physical equations [15] and experimental data [14], novel relations are developed to predict the fatigue life of UD CFRE. For the fatigue safety of UD CFRE, one thermal fatigue and three thermomechanical fatigue relations are provided. All these relations must be satisfied if a safe fatigue design is required. For deriving all the unknown parameters in these thermal and thermomechanical relations, fatigue experiments must be conducted. According to these relations, space temperature, ILSs, mechanical stress amplitude, and the number of thermal and mechanical cycles can affect the thermomechanical fatigue life of UD CFRE. However, these relations can be applied to predict the thermal and thermomechanical fatigue life of CNT/epoxy, if the parameters in these relations are adjusted to the CNT/epoxy properties.

As it is discussed in Section 3, thermally safe planets, moons, and asteroids can be identified with applying the thermal fatigue relation. Finally, according to the recent studies, CNT has a superior mechanical property when it is compared to CF. Therefore, it is expected to be applied in space composites such as CNT/epoxy in the future spacecrafts.

\section{Data Availability}

The data which have been used to write this manuscript are available in the following references: [2], [4], [5], [6], [14], [15], and [16].

\section{Conflicts of Interest}

The authors declare that they have no conflicts of interest.

\section{Acknowledgments}

Part of the funding of this research was provided by Professor Sanjeev Khanna, a faculty member of the Mechanical and Aerospace Engineering Department in the University of Missouri, Columbia. Therefore, the author would like to thank him for his support.

\section{References}

[1] B. Surowska, J. Bienias, and H. Debski, "Fatigue of unidirectional carbon fiber reinforced epoxy composites," in Proceedings of the ECCM15-15th European Conference on Composite Materials, pp. 24-28, Venice, Italy, June 2012.

[2] P. Coronado, A. Argüelles, J. Viña, V. Mollón, and I. Viña, "Influence of temperature on a carbon-fibre epoxy composite subjected to static and fatigue loading under mode-I delamination," International Journal of Solids and Structures, vol. 49, no. 21, pp. 2934-2940, 2012.

[3] A. Hosoi and H. Kawada, "Fatigue life prediction for transverse crack initiation of CFRP cross-ply and quasi-isotropic laminates," Materials, vol. 11, no. 7, p. 1182, 2018.

[4] F. Zhou, J. Zhang, S. Song, D. Yang, and C. Wang, "Effect of temperature on material properties of carbon fiber reinforced polymer (CFRP) Tendons: experiments and model assessment," Materials, vol. 12, no. 7, p. 1025, 2019.

[5] M. Mohamed, M. Johnson, and F. Taheri, "On the thermal fatigue of a room-cured Neat epoxy and its composite," Open Journal of Composite Materials, vol. 9, no. 2, pp. 145-163, 2019.

[6] M. Okayasu and Y. Tsuchiya, "Mechanical and fatigue properties of long carbon fiber reinforced plastics at low temperature," Journal of Science: Advanced Materials and Devices, vol. 4, no. 4, pp. 577-583, 2019.

[7] R. Prakash, "Significance of defects in the fatigue failure of carbon fibre reinforced plastics," Fibre Science and Technology, vol. 14, no. 3, pp. 171-181, 1981. 
[8] S. Singh and E. S. Greenhalgh, "Micromechanisms of interlaminar fracture in carbon-epoxy composites at multidirectional ply interfaces," in Proceedings of the 4th International Conference on Deformation and Fracture of Composites, pp. 201-210, Manchester, UK, March 1997.

[9] Y. Miyano, M. Nakada, and H. Kudoh, "Prediction of tensile fatigue life for unidirectional CFRP," in Progress in Durability Analysis of Composite Systems, pp. 303-308, CRC Press, Boca Raton, FL, USA, 1998.

[10] A. Sjogren and L. E. Asp, "Effects of temperature on delamination growth in a carbon/epoxy composite under fatigue loading," International Journal of Fatigue, vol. 24, no. 2-4, pp. 179-184, 2002.

[11] A. Argüelles, J. Viña, A. F. Canteli, M. A. Castrillo, and J. Bonhomme, "Interlaminar crack initiation and growth rate in a carbon-fibre epoxy composite under mode-I fatigue loading," Composites Science and Technology, vol. 68, no. 12, pp. 2325-2331, 2008.

[12] V. Mollón, J. Bonhomme, J. Viña, A. Argüelles, and A. Fernández-Canteli, "Influence of the principal tensile stresses on delamination fracture mechanisms and their associated morphology for different loading modes in carbon/ epoxy composites," Composites Part B: Engineering, vol. 43, no. 3, pp. 1676-1680, 2012.

[13] M. Okayasu, T. Yamazaki, K. Ota, K. Ogi, and T. Shiraishi, "Mechanical properties and failure characteristics of a recycled CFRP under tensile and cyclic loading," International Journal of Fatigue, vol. 55, pp. 257-267, 2013.

[14] S. Y. Park, H. S. Choi, W. J. Choi, and H. Kwon, "Effect of vacuum thermal cyclic exposures on unidirectional carbon fiber/epoxy composites for low earth orbit space applications," Composites Part B: Engineering, vol. 43, no. 2, pp. 726-738, 2012.

[15] A. Anvari, "Thermal life of carbon structures: from the earth to after the titan," International Journal of Aerospace Engineering, vol. 2018, Article ID 7628614, 6 pages, 2018.

[16] M. Chown, Solar System, Black Dog \& Leventhal Publishers, Inc, New York, NY, USA, 2011.

[17] S. Lijima, "Helical microtubules of graphic carbon," Nature, vol. 354 , no. 6348 , pp. 56-58, 1991.

[18] T. R. Fromy, F. K. Hanson, and T. Olsen, "The optimum dispersion of carbon nanotubes for epoxy nanocomposites: evolution of the particle size distribution by ultrasonic treatment," Journal of Nanotechnology, vol. 2012, Article ID 545930, 14 pages, 2012.

[19] J. Brown, T. Hajilounezhad, N. T. Dee, S. Kim, A. J. Hart, and M. R. Maschmann, "Delamination mechanics of carbon nanotube micropillars," ACS Applied Materials \& Interfaces, vol. 11, no. 38, pp. 35221-35227, 2019.

[20] T. Hajilounezhad, D. M. Ajiboye, and M. R. Maschmann, "Evaluating the forces generated during carbon nanotube forest growth and self-assembly," Materialia, vol. 7, p. 100371, 2019.

[21] T. Hajilounezhad and M. R. Maschmann, "Numerical investigation of internal forces during carbon nanotube forest self-assembly," in Proceedings of the International Mechanical Engineering Congress and Exposition, Pittsburgh, PA, USA, November 2018.

[22] Hajilounezhad T., Oraibi Z. A., Surya R., et al., Exploration of Carbon Nanotube Forest Synthesis-Structure Relationships Using Physics-Based Simulation and Machine Learning, 2019, IEEE, 1-8. 